\title{
On the Constructive Inverse Problem in Differential Galois Theory
}

\author{
Claude Mitschi* and Michael F. Singer ${ }^{\dagger}$
}

March 22, 2004

\begin{abstract}
We give sufficient conditions for a differential equation to have a given semisimple group as its Galois group. For any group $G=H \ltimes G^{0}, H$ finite and $G^{0}=\prod G_{i}$ where each $G_{i}$ is $\mathrm{SL}_{n_{i}}$ or $\mathrm{Sp}_{2 n_{i}}, n_{i} \geq 2$, we construct a differential equation over $C(x)$ having Galois group $G$.
\end{abstract}

\section{Introduction}

In [17, a large class of linear algebraic groups, including all groups with semisimple identity component, are shown to occur as Galois groups of differential equations $\frac{d Y}{d x}=A Y$ with $A$ an $n \times n$ matrix with coefficients in $C(x)$ where $C$ is an algebraically closed field of characteristic zero. The proof of this depended heavily on the analytic solution of the Riemann-Hilbert Problem and did not directly give a way of constructing such an equation $^{1}$. Techniques for constructing an equation with a given group have been produced for connected solvable groups [10, 11] and connected groups in general [13]. For groups that are not connected, the present authors showed in [14 that one could construct equations having any solvable-by-finite group as Galois group assuming one could produce (algebraic)

*Institut de Recherche Mathématique Avancée, Université Louis Pasteur et C.N.R.S., 7 rue René Descartes, 67084 Strasbourg Cedex, France, mitschi@math.u-strasbg.fr

${ }^{\dagger}$ North Carolina State University, Department of Mathematics, Box 8205, Raleigh, North Carolina 27695-8205, USA, singer@math.ncsu.edu. The second author would like to thank the Institut de Recherche Mathématique Avancée, Université Louis Pasteur et C.N.R.S., for its hospitality and support during the preparation of this paper. The preparation was also supported by NSF Grant CCR- 0096842.

${ }^{1}$ Once one knows that a group $G$ occurs as a differential Galois group of $C(x)$ for $C$ a recursive algebraically closed field of characteristic 0 , one can produce an equation for $G$ by listing all equations and using the algorithm from [5] to calculate an equation's Galois group to test until one is found. In this paper, we are interested in more direct methods. 
equations having any finite group as Galois group. In [4, Julia Hartmann has shown that any linear algebraic group can be realized as the Galois group of a linear differential equation over $C(x)$ and this proof shows that equations can be constructed once one knows how to construct equations for reductive groups (the proof uses the results of [17 in this case as well).

In this paper we will give a criterion, Proposition 3.2 for a differential equation to have a given semisimple algebraic group as a Galois group. We will use this proposition to show how one can construct differential equations with Galois group $G$, where $G=H \ltimes G^{0}, H$ finite and $G^{0}=\prod G_{i}$ with each $G_{i}$ equal to $\mathrm{SL}_{n_{i}}$ or $\mathrm{Sp}_{2 n_{i}}$. The rest of the paper is organized as follows. In Section 2, we discuss the criteria given in [14 and [4, that allow one to reduce the inverse problem for arbitrary groups over $C(x)$ to finding equivariant differential equations having connected Galois groups over an arbitrary finite Galois extension $K$ of $C(x)$. In Section 3, we develop the necessary group theory and give criteria for a differential equation to have a given semisimple group as its Galois group. In Section 4, we produce equations for the groups described above. In Section 5, we describe an alternate construction for groups of the form $\mathrm{SL}_{2} \ltimes W, W$ a finite group.

We wish to thank A. Bolibrukh and R. Schäfke for showing us how to select the local form of a differential equation so that Katz's Criterion ensures it is irreducible.

\section{Equivariant Equations}

In both 14] and 4, it was shown how to reduce the inverse problem for general groups to the inverse problem for groups of the form $H \ltimes G^{0}$, where $H$ is a finite group and $G^{0}$ is a connected group defined over $C$. We shall assume that we are given a finite extension $K$ of $C(x)$ with Galois group $H$ and we wish to find a Picard-Vessiot extension $E$ of $C(x)$ containing $K$ with Galois group $H \ltimes G^{0}$ such that the Galois action of $H \ltimes G^{0}$ on $K$ factors through the given action of $H$ on $K$. To attack this problem, these authors introduce the notion of an equivariant equation, which we now review.

Let $K$ be a finite Galois extension of $C(x)$ with Galois group $H$ and let $V$ be a vector space over $C$ that is also a right $H$-module. Notice that this also gives a right action of $H$ on $V(K)=K \otimes V$ given by $f \otimes v \mapsto f \otimes v \cdot h$ for $h \in H, f \in K$ and $v \in V$. We will again denote this action by $w \mapsto w h$ for $h \in H$ and $w \in V(K)$. The group $H$ can be seen to also act on the left on $V(K)$ via an action defined by $h(f \otimes v)=h f \otimes v$ for $f \in K$ and $v \in V$. We say that an element $w \in V(K)$ is equivariant if $h w=w h$ for all $h \in H$ (14], Definition 6.1; c.f. 4] Definition 3.5).

Let us now consider a semidirect product $G=H \ltimes G^{0}$ of the finite group $H$ and a connected linear algebraic group $G^{0}$ (defined over $C$ ) with multiplication given by $\left(h_{1}, g_{1}\right)\left(h_{2}, g_{2}\right)=$

$\left(h_{1} h_{2}, h_{2}^{-1} g_{1} h_{2} g_{2}\right)$. Let $\mathcal{G}$ be the Lie algebra of $G^{0}$. For any $h \in H$ the map $g \mapsto h^{-1} g h$ from 
$G^{0}$ to $G^{0}$ can be lifted to a map of $\mathcal{G}$ to $\mathcal{G}$ which we shall also denote by $A \mapsto h^{-1} A h$. In this way we may consider $\mathcal{G}$ as a right $H$-module. With this convention, we may speak of equivariant elements of $\mathcal{G}(K)$. In concrete terms, an element $A \in \mathcal{G}(K)$ is equivariant if, for any $h \in H$, the result of applying $h$ (as an element of the Galois group of K) to the entries og $A$ is the same as conjugating $A$ by $h^{-1}$ (where $h$ is now considered as an element of $G)$. We will say a differential equation $Y^{\prime}=A Y$ is equivariant if $A \in \mathcal{G}(K)$ is equivariant. We will throughout the paper use the notation $Y^{\prime}$ for $\partial Y$, where $\partial$ is the unique extension of $\frac{d}{d x}$ on $K$. Using this notion of equivariance, we have the following criterion (c.f., 14] Proposition 6.3; 4, Proposition 3.10):

Proposition 2.1 Let $G$ and $K$ be as above and let $A$ be an equivariant element of $\mathcal{G}(K)$ such that the Picard-Vessiot extension $E$ of $K$ corresponding to the equation $Y^{\prime}=A Y$ has Galois group $G^{0}$. Then $E$ is a Picard-Vessiot extension of $C(x)$ with Galois group $G$.

To apply this result, we will need ways of constructing equivariant elements $A$ of $\mathcal{G}(K)$ and criteria to ensure that the equation $Y^{\prime}=A Y$ has the desired Galois group over $K$. The remainder of this section is devoted to the first task and the next section to the second task. In the following, we shall think of $C$ as embedded in the complex numbers and denote by $C\{t\}$ the subring of convergent power series of $C[[t]]$, and by $C(\{t\})$ its quotient field $C(\{t\})$.

Lemma 2.2 Let $\pi: \mathbf{C} \rightarrow \mathbf{P}^{1}$ be a covering of the projective line by a curve $\mathbf{C}$ with function field $K$, such that $C(x) \subset K$ is induced by $\pi$. There exists a computable set of points $\mathcal{S} \subset \mathbf{P}^{1}$ such that the following is true: Given

1. an integer $M$,

2. points $p_{1}, \ldots p_{r} \in \mathbf{C}$ with $\pi\left(p_{i}\right) \notin \mathcal{S}$ and $\pi\left(p_{i}\right) \neq \pi\left(p_{j}\right)$ for $i \neq j$,

3. local parameters $t_{i}$ at $p_{i}$ and

4. elements $A_{1}, \ldots, A_{r} \in \mathcal{G}(C)$,

there exists an equivariant $A \in \mathcal{G}(K)$ such that at each $p_{i}$, we have

$$
A=A_{i} t^{M}+t^{M+1}\left(B_{i}(t)\right)
$$

where $t=t_{i}$ and $B_{i}(t) \in \mathcal{G}(C\{t\})$.

Proof. We can consider $\mathcal{G}(C)$ as a left $H$-module under the action $v \mapsto h v h^{-1}$ for any $h \in H$. We define an action of $H$ on $\mathcal{G}(K)$ by the formula $h(f \otimes v)=h(f) \otimes h v h^{-1}$. This action satisfies $h(a w)=h(a) h(w)$ for all $h \in H, a \in K, w \in \mathcal{G}(K)$ and so, by a result of Kolchin and Lang (12, Exercises 31 and 32, p. 550), one can construct an invariant basis of $\mathcal{G}(K)$ over $K$, that is, a basis $\tilde{e}_{1}, \ldots, \tilde{e}_{s}$ such that $h\left(\tilde{e}_{i}\right)=\tilde{e}_{i}$ for $i=1, \ldots, s$. This basis 
is an equivariant basis in the above sense, that is $h \tilde{e}_{i}=\tilde{e}_{i} h$ for all $h \in H$. Fix a basis $e_{1}, \ldots, e_{s}$ of $\mathcal{G}(C)$ and define $B \in \mathrm{GL}_{s}(K)$ such that $\left(\tilde{e}_{1}, \ldots, \tilde{e}_{s}\right)=\left(e_{1}, \ldots, e_{s}\right) B$. For any $f_{1}, \ldots, f_{s} \in C(x), \sum_{i=1}^{s} f_{i} \tilde{e}_{i}$ is an equivariant element of $\mathcal{G}(K)$. We shall now show how one can select the $f_{i}$ so that the conclusions of the Lemma are satisfied. Let $\mathcal{S}$ be the image under $\pi$ of those points $p \in \mathbf{C}$ satisfying at least one of the following conditions:

1. $p$ is a singular point of $\mathbf{C}$ or is a ramification point of $\pi$, or

2. $p$ is a pole of an entry of $B$, or

3. $\left\{\tilde{e}_{1}(p), \ldots, \tilde{e}_{s}(p)\right\}$ fails to be a basis of $\mathcal{G}(K)$, i.e., $\operatorname{det}(B(p))=0$.

Note that condition 1. implies that we may select $t=x-\pi(p)$ to be a local coordinate for any point $p$ with $\pi(p) \notin \mathcal{S}$, if $\pi(p)$ is finite, and $t=1 / x$ if $\pi(p)$ is infinite. We shall use these local coordinates. From these conditions, we see that at each $p_{i}$ with $\pi\left(p_{i}\right) \notin \mathcal{S}$, there exist $c_{i, j} \in C$ such that $A_{i}=\sum_{j=1}^{s} c_{i, j} \tilde{e}_{j}\left(p_{i}\right)$. Let $f_{j} \in C(x)$ satisfy $f_{j}=c_{i, j} t^{M}+t^{M+1} b_{i, j}$ where $b_{i, j} \in C\{t\}$ when written in local coordinates $t=t_{j}$ at the point $p_{j}$. We then have that $A=\sum_{j=1}^{s} f_{j} \tilde{e}_{j}$ satisfies the conclusion of the Lemma.

Corollary 2.3 Let $\pi: \mathbf{C} \rightarrow \mathbf{P}^{1}$ be a covering of the projective line by a curve $\mathbf{C}$ with function field $K$ such that $C(x) \subset K$ is induced by $\pi$. There exists a computable set of points $S \subset \mathbf{P}^{1}$ such that the following is true: Given

1. integers $M<N$,

2. points $p_{1}, \ldots p_{r} \in \mathbf{C}$ with $\pi\left(p_{i}\right) \notin \mathcal{S}$ and $\pi\left(p_{i}\right) \neq \pi\left(p_{j}\right)$ for $i \neq j$,

3. local parameters $t_{i}$ at $p_{i}$ and

4. for each $i=1, \ldots$, r elements $A_{i, M}, \ldots, A_{i, N} \in \mathcal{G}(C)$,

there exists an equivariant $A \in \mathcal{G}(K)$ such that at each $p_{i}$, we have

$$
A=A_{i, M} t^{M}+\ldots+A_{i, N} t^{N}+t^{N+1}\left(B_{i}(t)\right)
$$

where $t=t_{i}$ and $B_{i}(t) \in \mathcal{G}(C\{t\})$

Proof. Let $\mathcal{S}$ be as before. We will proceed by induction on $N-M$. Assume that we have found an equivariant $A_{0} \in \mathcal{G}(K)$ such that at each $p_{i}$, we have

$$
A_{0}=A_{i, M} t^{M}+\ldots+A_{i, N-1} t^{N-1}+t^{N}\left(B_{i}(t)\right)
$$

where $t=t_{i}$ and $B_{i}(t) \in \mathcal{G}(C\{t\})$. Let $C_{i}$ be the coefficient of $t^{N}$ in the expansion of $A_{0}$ at $p_{i}$. Using Lemma 2.2. we can find an equivariant $\tilde{A} \in \mathcal{G}(K)$ such that

$$
\tilde{A}=\left(A_{i, N}-C_{i}\right) t^{N}+t^{N+1}\left(\tilde{B}_{i}(t)\right)
$$

where $t=t_{i}$ and $\tilde{B}_{i}(t) \in \mathcal{G}(C\{t\})$. The element $A=A_{0}+\tilde{A}$ satisfies the conclusion of the Corollary. 
Example 2.4 The group $\mathrm{Z} / 2 \mathrm{Z} \ltimes \mathrm{SL}_{2}$

We shall illustrate the above results for this group where $h=-1 \in \mathbf{Z} / 2 \mathbf{Z}$ acts on $\mathrm{SL}_{2}$ by sending a matrix to the transpose of its inverse. Note that the action of this element on $\mathrm{sl}_{2}$ sends an matrix to the negative of its transpose. Let $K=C(x, \sqrt{x})$ with Galois group $H$. The elements

$$
\begin{aligned}
\tilde{e}_{1} & =\left(\begin{array}{cc}
\sqrt{x} & 0 \\
0 & -\sqrt{x}
\end{array}\right) \\
\tilde{e}_{2} & =\left(\begin{array}{cc}
0 & \sqrt{x} \\
\sqrt{x} & 0
\end{array}\right) \\
\tilde{e}_{3} & =\left(\begin{array}{cc}
0 & -1+\sqrt{x} \\
1+\sqrt{x} & 0
\end{array}\right)
\end{aligned}
$$

form an equivariant basis of $\operatorname{sl}_{2}(K)$. We now will construct an equivariant element $A$ of $\mathcal{G}(K)$ with the following prescribed principal parts at the points $(4,2),(9,3)$ and $(16,4)$ of the curve $y^{2}-x=0$ (we will see in Section 4 that the equation $Y^{\prime}=A Y$ is then an equivariant equation with Galois group $\mathrm{SL}_{2}$ over $K$ ).

$$
\begin{aligned}
& \text { At } p_{0}=(4,2), \text { with } t=x-4, A=\frac{\left(\begin{array}{ll}
0 & 0 \\
1 & 0
\end{array}\right)}{t^{2}}+\frac{\left(\begin{array}{ll}
0 & 1 \\
0 & 0
\end{array}\right)}{t} \text { terms involving } t^{j}, j \geq 0 \\
& \text { At } p_{1}=(9,3), \text { with } t=x-9, A=\frac{\left(\begin{array}{cc}
\sqrt{2} & 0 \\
0 & -\sqrt{2}
\end{array}\right)}{t}+\text { terms involving } t^{j}, j \geq 0 \\
& \text { At } p_{2}=(16,4), \text { with } t=x-16, A=\frac{\left(\begin{array}{ll}
0 & 0 \\
1 & 0
\end{array}\right)}{t}+\text { terms involving } t^{j}, j \geq 0
\end{aligned}
$$

A calculation shows that the following rational functions yield the desired result for $A:=$ $f_{1} \tilde{e}_{1}+f_{2} \tilde{e}_{2}+f_{3} \tilde{e}_{3}$ :

$$
\begin{aligned}
& f_{1}=-\frac{\sqrt{2}}{105} \frac{(x-4)(x-16)}{x-9} \\
& f_{2}=-\frac{1}{240} \frac{(x-9)(x-16)}{(x-4)^{2}}+\frac{311}{28800} \frac{(x-9)(x-16)}{x-4}-\frac{3}{672} \frac{(x-9)(x-4)}{x-16} \\
& f_{3}=\frac{1}{120} \frac{(x-9)(x-16)}{(x-4)^{2}}-\frac{43}{7200} \frac{(x-9)(x-16)}{x-4}+\frac{1}{168} \frac{(x-9)(x-4)}{x-16}
\end{aligned}
$$




\section{Group Theory and its Differential Consequences}

Throughout this section $H$ stands for any (not necessarily finite) subgroup of $G$. The principal tool that we shall use is the following result:

Lemma 3.1 Let $G \subset \mathrm{GL}_{n}(C)$ be a connected semisimple algebraic group of rank $r$ with Lie algebra $\mathcal{G}$ and let $\mathrm{Ad}: G \rightarrow \mathrm{GL}(\mathcal{G})$ be the adjoint representation. Let $H$ be an algebraic subgroup of $G$ and assume:

1. $H$ acts reductively on $\mathcal{G}$ via the adjoint representation.

2. $H$ contains an element having at least $r$ multiplicatively independent eigenvalues.

3. $H$ contains an element $u$ such that $A d(u)$ is a unipotent element with an $r$ dimensional eigenspace corresponding to the eigenvalue 1.

Then $H=G$.

Proof. Let $\mathcal{H}$ be the Lie algebra of $H$. It is enough to show that $\mathcal{H}=\mathcal{G}$. To do this we will use the following result ([3], p. 246, Ex. 5): If $\mathcal{G}$ is a semisimple Lie algebra and $\mathcal{H}$ is a subalgebra acting reductively on $\mathcal{G}$ via the adjoint representation, of the same rank as $\mathcal{G}$, and containing a principal $\mathrm{sl}_{2}$-triple of $\mathcal{G}$, then $\mathcal{G}=\mathcal{H}$. We shall show that each of the conditions of the Lemma implies the corresponding condition of this latter result.

If $H$ acts reductively on $\mathcal{G}$ then so does $\mathcal{H}$.

Let $h$ be an element of $H$ having $r$ multiplicatively independent eigenvalues. Then $h^{i}$ has the same property for all $i>0$, so we may assume that $h \in H^{0}$. We may write $h=h_{s} h_{u}$ where $h_{s}$ and $h_{u}$ are the semisimple and unipotent parts of $h$, respectively. Since the eigenvalues of $h$ and $h_{s}$ coincide and $h_{s} \in H^{0}$, we may assume that $h$ is semisimple and that $h$ lies in some maximal torus $T$ of $H$. We may assume that $T$ is a subgroup of diagonal matrices and that the $r$ multiplicatively independent eigenvalues of $h$ are the first $r$ entries on the diagonal of $h$. If $\chi_{i}$ denotes the character that picks out the $i^{\text {th }}$ element on the diagonal, we see that no nontrivial power product of $\chi_{1}, \ldots, \chi_{r}$ is trivial on $h$. Therefore $\chi_{1}, \ldots, \chi_{r}$ are multiplicatively independent on $T$ and so the dimension of $T$ is greater than or equal to $r$, that is, the rank of $\mathcal{H}$ is $r$.

Let $u \in H$ satisfy the property that $\operatorname{Ad}(u)$ is unipotent with an $r$-dimensional eigenspace corresponding to the eigenvalue 1 . Since $\operatorname{Ad}(u)=\operatorname{Ad}\left(u_{s}\right) \operatorname{Ad}\left(u_{u}\right)$ where $u_{s}$ and $u_{u}$ are semisimple and unipotent, we have $\operatorname{Ad}\left(u_{s}\right)=1$ and can replace $u$ with $u_{u}$ and assume that $u$ is unipotent. If we let $n=\log (u)$ we have that $\{\exp ($ an $) \mid a \in C\}$ is the unique smallest closed subgroup of $H$ containing $u$ and that $n \in \mathcal{H}$ (see Lemma C, p. 96 and Exercise 
11, p. 101 of [6]). This implies that $\operatorname{ad}(n)=\log (\operatorname{Ad}(u))$ and so a calculation implies that the dimension of the nulspace of $\operatorname{ad}(n)$ is $r$. Since $n$ is nilpotent, the Jacobson-Morozov Theorem ( 3 p. 162) implies that $n$ is contained in an $\mathrm{sl}_{2}$-triple in $\mathcal{H}$ and furthermore this triple is principal in $\mathrm{SL}_{n}$ ([3], p. 166).

The above lemma gives us the following criterion to ensure that a differential equation has a given semisimple group as its Galois group.

Proposition 3.2 Let $G \subset \mathrm{GL}_{n}(C)$ be a connected semisimple linear algebraic group of rank $r$ with Lie algebra $\mathcal{G}$ and $\mathbf{C}$ a curve with function field $K \supset C(x)$. Let $Y^{\prime}=A Y$ be a differential equation with $A \in \mathcal{G}(K)$. Let $H$ be the Galois group of $Y^{\prime}=A Y$ over $K$ and assume that

1. $H$ is reductive,

2. There exists a point $p_{1} \in C$ such that in terms of some local coordinate $t$ at $p_{1}$, we have that

$$
\frac{d Y}{d t}=\left(\frac{A_{1}}{t}+B_{1}(t)\right) Y
$$

where $A_{1}$ is semisimple and has $r$ eigenvalues that are $\mathbf{Z}$-independent $\bmod \mathbf{Z}$ and $B_{1}(t) \in C\{t\}$.

3. There exists a point $p_{2} \in C$ such that in terms of some local coordinate $t$ at $p_{2}$, we have that

$$
\frac{d Y}{d t}=\left(\frac{A_{2}}{t}+B_{2}(t)\right) Y
$$

where $\operatorname{ad}\left(A_{2}\right)$ is nilpotent with a kernel of dimension $r$ and $B_{2}(t) \in C\{t\}$.

Then $H=G$.

Proof. We know that $H$ is an algebraic subgroup of $G$ (c.f., 14 Prop.2.1). We shall show that it satisfies the hypotheses of Lemma 3.1. Clearly hypothesis 1 . is satisfied.

To see that hypothesis 2. of Lemma 3.1 is satisfied, note that the present hypothesis 2 . implies that the distinct eigenvalues of $A_{1}$ do not differ by integers. This implies that the equation $Y^{\prime}=A Y$ is equivalent (over $C((t))$ and even $C(\{t\})$ ) to the equation $\frac{d Y}{d t}=\frac{A_{1}}{t} Y$ whose monodromy at $p_{1}$ is given by $e^{2 \pi i A_{1}}$ (see Section 3 of [1] or Sections 3.3. and 5.1.1 of [16]). Note that this element has at least $r$ multiplicatively independent eigenvalues. Since $K$-equivalent differential equations have conjugate Galois groups there exists an element $h$ of $H$ which is conjugate (in $\mathrm{GL}_{n}(C)$ ) to $e^{2 \pi i A_{1}}$, and hence $h$ satisfies hypothesis 2 . of Lemma 3.1 .

To verify hypothesis 3 we must argue in a more careful way. For this we use the results 
of Section 8 of [1]. Since $\operatorname{ad}\left(A_{2}\right)$ is nilpotent, the spectral subspaces $\mathbf{g}_{\lambda}$ of $\operatorname{ad}\left(A_{2}\right)$ corresponding to all positive integers $\lambda$ are zero (c.f., Section 8.5 of [1]). Therefore Proposition 8.5 of [1] implies that there exists a $g \in G(C\{t\})$ such that the gauge transform $Y=g Z$ yields an equation of the form $\frac{d Z}{d t}=\frac{\tilde{A_{2}}}{t} Z$, where $\operatorname{ad}\left(\tilde{A}_{2}\right)$ is again nilpotent with a kernel of dimension $r$. This equation has solution $z=e^{\tilde{A_{2}} \log t}$ and local monodromy (at $\left.p_{2}\right)$ given by $z \mapsto e^{2 \pi i \tilde{A}_{2}} z$. Therefore the solution $y=g z \in G(C\{t\})$ of the equation $Y^{\prime}=A Y$ has local monodromy $g e^{2 \pi i A_{2}} g^{-1}$, that is, there is an $u \in H$ which is conjugate in $G(C)$ to $e^{2 \pi i \tilde{A}_{2}}$. One now sees that $\operatorname{Ad}(u)$ has the desired property.

From Section 2 it is clear that we should have no trouble fulfilling hypotheses 2. and 3. of Proposition [3.2. The difficulty arises in trying to ensure that hypothesis 1 . is satisfied. Before we describe criteria that ensure this hypothesis is satisfied, we will recall the facts we need relating the local Galois groups and the global Galois group.

Let $\pi: \mathbf{C} \rightarrow \mathbf{P}^{1}$ be a nonsingular curve over the projective line and let $C(x) \subset K$ be the corresponding inclusion of function fields. Let $p \in \mathbf{C}$ and assume that $\mathbf{C}$ is not ramified at $p$ and that $\pi(p) \neq \infty$. What follows can be developed without these assumptions but they simplify the exposition and will hold in our applications. We can then embed $K$ into $C((t)), t=x-\pi(p)$, by expanding each element of $K$ as a series in $t$. We shall identify $K$ with its image and write $K \subset C((t))$. In fact, we have that $K \subset C(\{t\}) \subset C((t))$. Any differential equation $Y^{\prime}=A Y, A \in \operatorname{gl}(K)$ can be considered as a differential equation over $C((t))$ and so we can form a Picard-Vessiot extension $E$ of $C((t))$ corresponding to this equation. Let $y$ be a fundamental solution matrix of $Y^{\prime}=A Y$ having entries in $E$. If we denote by $K(y)$ and $C(\{t\})(y)$ the fields generated by the entries of $y$ over $K$ and $C(\{t\})$ respectively, we see that $K(y)$ is a Picard-Vessiot extension for $Y^{\prime}=A Y$ over $K$ and $C(\{t\})(y)$ is a Picard-Vessiot extension for this equation over $C(\{t\})$. We denote by $G$, $G_{\text {conv }}$ and $G_{\text {form }}$ the Galois groups of $K(y)$ over $K, C(\{t\})(y)$ over $C(\{t\})$ and $E$ over $C((t))$ respectively. One then easily checks that there are natural injections $G_{\text {form }} \hookrightarrow G_{\text {conv }} \hookrightarrow G$ and that the actions of the former two groups on the solution space of the differential equation coincide with their actions as embedded subgroups of $G$. These considerations lead to:

Lemma 3.3 Let $\mathbf{C}$ be a curve with function field $K \supset C(x)$ and let $Y^{\prime}=A Y$ be a differential equation with $A \in \mathcal{G}(K)$. If there exists a point $p \in \mathbf{C}$ as above such that the equation is irreducible over $C(\{t\})$, then the equation is irreducible over $K$ and its Galois group is reductive. In particular, if it is irreducible over $C((t))$, then the Galois group of $Y^{\prime}=A Y$ over $K$ is reductive.

Proof. A differential equation is irreducible over a differential field with algebraically closed field of constants if and only if its Galois group acts irreducibly on the solution space of the equation in its Picard-Vessiot extension. If $Y^{\prime}=A Y$ is irreducible over $C(\{t\})$ then $G_{\text {conv }}$ acts irreducibly on the solutions space. Since $G_{c o n v} \hookrightarrow G$, we have that $G$ acts 
irreducibly on this space and so the equation is irreducible over $K$. We can furthermore conclude that $G$ is reductive since it has an irreducible faithful representation. The final statement follows in a similar manner.

It is much easier to show that a differential equation is irreducible over $C((t))$ than to show it is irreducible over $C(\{t\})$. Regrettably, from our point of view, irreducibility over $C((t))$ puts severe restrictions on the Galois group $G$ of $Y^{\prime}=A Y$ over $C(x)$. One can deduce from (16], Remark 3.34) that if $Y^{\prime}=A Y$ is irreducible over $C((t))$, then

1. the identity component $G_{\text {form }}^{0}$ of $G_{\text {form }}$ is a (connected) torus,

2. as a $G_{\text {form }}^{0}$-module, the solution space is the sum of one dimensional invariant subspaces corresponding to distinct characters of $G_{\text {form }}^{0}$, and

3. there is an element $\gamma \in G_{\text {form }}$ whose action on $G_{\text {form }}^{0}$ by conjugation cyclically permutes these characters of $G_{\text {form }}^{0}$.

In ([7, 3.2.9 and 3.2.8), Katz has shown that a connected algebraic subgroup $G$ of $\mathrm{SL}_{n}$, containing a closed subgroup $H \subset G$ satisfying the properties of $G_{\text {form }}$ above must be of the form $\prod G_{i}$ where each $G_{i}$ is either $\mathrm{SL}_{n_{i}}$ or $\mathrm{Sp}_{n_{i}}, n_{i}$ even in the latter case, and the $n_{i}$ are pairwise relatively prime. Katz further shows that the $n$-space (in our case the solution space) can be written as a tensor product $\otimes V_{i}$ of representations of these groups where each $V_{i}$ is the standard or contragredient representation of $G_{i}$ if $G_{i}=\mathrm{SL}_{n_{i}}$ or the standard representation of $G_{i}$ if $G_{i}=\mathrm{Sp}_{n_{i}}$.

Nonetheless, Lemma 3.3 together with Proposition 3.2 will allow us to construct equations $Y^{\prime}=A Y$ having Galois group $\mathrm{SL}_{n}$ or $\mathrm{Sp}_{2 n}$. These two results yield the following criteria:

Proposition 3.4 Let $G \subset \mathrm{SL}_{n}(C)$ be a connected simple linear algebraic group of rank $r$ with Lie algebra $\mathcal{G}$ and $\mathbf{C}$ a curve with function field $K \supset C(x)$. Let $Y^{\prime}=A Y$ be a differential equation with $A \in \mathcal{G}(K)$. Let $H$ be the Galois group of $Y^{\prime}=A Y$ over $K$ and assume that

1. There exists a point $p_{0} \in \mathbf{C}$ such the equation $Y^{\prime}=A Y$ has a unique slope of the form $\frac{a}{n},(a, n)=1$.

2. There exists a point $p_{1} \in \mathbf{C}$ such that in terms of some local coordinate $t$ at $p_{1}$, we have that

$$
\frac{d Y}{d t}=\left(\frac{A_{1}}{t}+B_{1}(t)\right) Y
$$

where $A_{1}$ is semisimple and has $r$ eigenvalues that are $\mathbf{Z}$-independent $\bmod \mathbf{Z}$ and $B_{1}(t) \in \operatorname{gl}(C\{t\})$. 
3. There exists a point $p_{2} \in \mathbf{C}$ such that in terms of some local coordinate $t$ at $p_{2}$, we have that

$$
\frac{d Y}{d t}=\left(\frac{A_{2}}{t}+B_{2}(t)\right) Y
$$

where $\operatorname{ad}\left(A_{2}\right)$ is nilpotent with a kernel of dimension $r$ and $B_{2}(t) \in \operatorname{gl}(C\{t\})$.

Then $H=G$. Furthermore, if this is the case, then $G$ must be either $\mathrm{SL}_{n}$ or $\mathrm{Sp}_{2 n}$.

Proof. We refer to [1], [7] or [16] for the definition and properties of the slopes of a differential equation at a singular point. From (2.2.8) of [7] or Remark 3.34 of [16], one sees that hypothesis 1. above implies hypothesis 1. of Proposition 3.2. The last statement follows from the discussion preceding the statement of this proposition.

\section{Equivariant Equations for $G^{0}=\Pi G_{i}$ with $G_{i}=\mathrm{SL}_{n_{i}}$ or $G_{i}=\mathrm{Sp}_{2 n_{i}}$}

In this section $G$ is a given linear algebraic group with identity component $G^{0}$ and $H$, as in section 2, denotes a finite subgroup of $G$. Let $K$ be a Galois extension of $C(x)$ with Galois group $H$. We will begin by showing how one can construct equivariant equations $Y^{\prime}=$ $A Y, A \in \mathcal{G}(K)$ where $\mathcal{G}=\mathrm{sl}_{n}$ (respectively $\mathcal{G}=\mathrm{sp}_{2 n}, n \geq 2$ ) having Galois group $G^{0}=\mathrm{SL}_{n}$ (resp. $G^{0}=\operatorname{Sp}_{2 n}$ ) over $K$. To do this, we will choose an element $A \in \mathcal{G}(K)$ satisfying the hypotheses of Proposition 3.4. We begin by defining four matrices in $\mathcal{G}(C)$, which we will assume is $\mathrm{sl}_{n}$ (resp. $\mathrm{sp}_{2 n}$ as a subalgebra of $\mathrm{sl}_{2 n}$ via its standard representation).

$A_{0,1}, A_{0,2}$ : Let $A_{3}=\left(a_{i, j}\right)$ be the matrix defined by $a_{i+1, i}=1$ for $i=0, \ldots, n-1$ and $\overline{a_{i, j}=0 \text { if }} j+1 \neq i$ and let $A_{4}$ be the matrix with 1 as the $(1, n)$ entry and 0 everywhere else. If $G^{0}=\mathrm{SL}_{n}$, let $A_{0,1}=A_{3}$ and $A_{0,2}=A_{4}$. If $G^{0}=\mathrm{Sp}_{2 n}$, let

$$
A_{0,1}=\left(\begin{array}{cc}
A_{3} & 0 \\
A_{4} & -A_{3}
\end{array}\right) \text { and } A_{0,2}=\left(\begin{array}{cc}
0 & (-1)^{n} A_{4} \\
0 & 0
\end{array}\right) \text {. }
$$

Note that in both cases the matrix $A_{0,1}+A_{0,2}$ is a matrix whose eigenvalues are the $n^{\text {th }}$ roots of 1 in the case of $\mathrm{SL}_{n}$ and the $2 n^{\text {th }}$ roots of 1 in the case of $\mathrm{Sp}_{2 n}$.

$\underline{A_{1}}$ : Let $r_{1}, \ldots, r_{n-1} \in C$ be $\mathbf{Z}$-linearly independent $\bmod \mathbf{Z}$ and let $r_{n}=-\sum r_{i}$. If $G^{0}=\mathrm{SL}_{n}$ let $A_{1}=\operatorname{diag}\left(r_{1}, \ldots, r_{n}\right)$, the diagonal matrix of trace zero with the $r_{i}$ on the diagonal. If $G^{0}=\operatorname{Sp}_{2 n}$, let $A_{1}=\operatorname{diag}\left(\operatorname{diag}\left(r_{1}, \ldots, r_{n}\right),-\operatorname{diag}\left(r_{1}, \ldots, r_{n}\right)\right)$.

$\underline{A_{2}}$ : Any semisimple Lie algebra of rank $r$ contains principal nilpotent elements $u$ ([3], Proposition 8, p. 166). These can be constructed by decomposing the algebra as the sum of a Cartan algebra and nonzero root spaces and letting $u=\sum_{\alpha \in \Phi^{+}} v_{\alpha}$ where $\Phi^{+}$is the set of positive roots and each $v_{\alpha}$ is nonzero (3], Proposition 10, p. 168). For example, in $\mathrm{sl}_{n}$ 
we can take the matrix $u=\left(a_{i, j}\right)$ where $a_{i, j}=0$ if $i \geq j$ and $a_{i, j}=1$ if $i<j$. We let $A_{2}$ be such an element.

We now will apply Corollary 2.3. Select 3 points $p_{0}, p_{1}$, and $p_{2}$ whose projections are distinct and not in $\mathcal{S}$. Corollary 2.3 implies that one can produce an $A \in \mathcal{G}(K)$ such that in terms of the local coordinate $t$ at these points, the equation $Y^{\prime}=A Y$ has the following form:

$$
\begin{aligned}
& \text { At } p_{0}, \frac{d Y}{d t}=\left(\frac{A_{0,1}}{t^{2}}+\frac{A_{0,2}}{t}+\text { terms involving } t^{j}, j \geq 0\right) Y . \\
& \text { At } p_{1}, \frac{d Y}{d t}=\left(\frac{A_{1}}{t}+\text { terms involving } t^{j}, j \geq 0\right) Y . \\
& \text { At } p_{2}, \frac{d Y}{d t}=\left(\frac{A_{2}}{t}+\text { terms involving } t^{j}, j \geq 0\right) Y .
\end{aligned}
$$

We now will check that the conditions of Proposition 3.4 hold. To see that there is a unique slope at $p_{0}$, let us assume that $\mathcal{G}=\mathrm{sl}_{n}$ and let $g=\operatorname{diag}\left(1, t^{1 / n}, t^{2 / n}, \ldots, t^{n-1 / n}\right)$. Note that for any matrix $\left(a_{i, j}\right)$, we have that

$$
g\left(a_{i, j}\right) g^{-1}=\left(t^{\frac{i-j}{n}} a_{i, j}\right)
$$

Therefore

$$
g[A]=g A g^{-1}+g^{\prime} g^{-1}=\frac{A_{0,1}+A_{0,2}}{t^{2-\frac{1}{n}}}+\text { terms involving } t^{j}, j \geq 2-\frac{1}{n} .
$$

This is usually called a shearing-transform of the equation $\frac{d Y}{d t}=A Y$. Since the matrix $A_{0,1}+A_{0,2}$ is semisimple, there will be a unique slope, equal to $2-1 / n$ ([1], Proposition 4.2 and the subsequent paragraphs). Therefore, as noted before, the equation will be irreducible over $C((t))$. If $\mathcal{G}=\mathrm{sp}_{2 n}$, we let $g=\operatorname{diag}\left(1, t^{1 / 2 n}, \ldots, t^{2 n-1 / 2 n}\right)$ and proceed as above.

Finally, at $p_{1}$ and $p_{2}$ the required conditions are obviously satisfied. Therefore, the equivariant equation $Y^{\prime}=A Y$ has Galois group $G^{0}$ over $K$ and so using the techniques of [14] or 4 one can construct an equation having Galois group $H \ltimes G^{0}$ over $C(x)$. In particular, the example given in Section 2 was constructed in the above manner and so has Galois group $\mathrm{SL}_{2}$ over $K=C(x, \sqrt{x}$ ) (another example with this group is given via an ad hoc construction in [4], p. 42.)

We now turn to the general situation where $G^{0}=\prod_{i=1}^{m} G_{i}, G_{i}=\mathrm{SL}_{n_{i}}$ or $G_{i}=\mathrm{Sp}_{2 n_{i}}$. Let $\mathcal{G}_{i}$ denote the Lie algebra of $G_{i}$ and $\mathcal{G}=\oplus_{i=1}^{m} \mathcal{G}_{i}$. Let $\rho_{i}: G_{i} \rightarrow G^{0}$ and $\pi_{i}: G^{0} \rightarrow G_{i}$ denote the canonical injections and projections respectively. We will denote by $d \rho_{i}: \mathcal{G}_{i} \rightarrow \mathcal{G}$ and $d \pi_{i}: \mathcal{G} \rightarrow \mathcal{G}_{i}$ the corresponding maps on the level of Lie algebras. Let $\left\{p_{0}^{i}, p_{1}^{i}, p_{2}^{i}\right\}_{i=1}^{m}$ be $3 m$ points on $\mathbf{C} \backslash \mathcal{S}$ having distinct projections. For each $i$, we denote by $A_{0,1}^{i}, A_{0,2}^{i}, A_{1}^{i}, A_{2}^{i} \in \mathcal{G}_{i}$ be matrices as above. Corollary 2.3 implies that one can produce an $A \in \mathcal{G}(K)$ such that 
in terms of the local coordinate $t$ at these points, the equation $Y^{\prime}=A Y$ has the following form:

$$
\begin{aligned}
& \text { At } p_{0}^{i}, \frac{d Y}{d t}=\left(\frac{d \rho_{i}\left(A_{0,1}^{i}\right)}{t^{2}}+\frac{d \rho_{i}\left(A_{0,2}^{i}\right)}{t}+\text { terms involving } t^{j}, j \geq 0\right) Y . \\
& \text { At } p_{1}^{i}, \frac{d Y}{d t}=\left(\frac{d \rho_{i}\left(A_{1}\right)}{t}+\text { terms involving } t^{j}, j \geq 0\right) Y . \\
& \text { At } p_{2}^{i}, \frac{d Y}{d t}=\left(\frac{d \rho_{i}\left(A_{2}\right)}{t}+\text { terms involving } t^{j}, j \geq 0\right) Y .
\end{aligned}
$$

Let $A_{i}=d \pi_{i}(A)$. We then have that the differential equations $Y^{\prime}=A_{i} Y$ are non-singular at each $p_{0}^{j}, p_{1}^{j}, p_{2}^{j}$ for $j \neq i$ and expand as equations (11), (2), (3) at $p_{0}^{i}, p_{1}^{i}, p_{2}^{i}$. These two facts will be the key to showing that $Y^{\prime}=A Y$ has Galois group $G$.

Let $E$ be the Picard-Vessiot extension of $K$ corresponding to $Y^{\prime}=A Y$. Since $A \in \mathcal{G}(K)$, the proof of Proposition 1.31 of [16] shows that we can assume that $K$ is generated by the entries of an element $g \in G^{0}(E)$ such that $g^{\prime}=A g$. Writing $g=\left(g_{1}, \ldots, g_{m}\right)$ where each $g_{i} \in G_{i}$ and $A=\left(A_{1}, \ldots, A_{m}\right)$ where each $A_{i} \in \mathcal{G}(K)$, we have that $g_{i}^{\prime}=A_{i} g_{i}$ and so $E$ contains the Picard-Vessiot extension $E_{i}=K\left(g_{i}\right)$ of $K$ corresponding to each of the equations. From the discussion at the beginning of this section, we know that the Galois group of $Y^{\prime}=A_{i} Y$ over $K$ is $G_{i}$. We shall now show that the Galois group $G^{\prime}$ of $Y^{\prime}=A Y$ over $K$ is $G$.

Since $A \in \mathcal{G}(K)$, we have that $G^{\prime} \subset G$ ([16], Proposition 1.31). Assume that $G^{\prime} \neq G$. We will show that this implies that there exist indices $i \neq j$ such that $E_{i}$ lies in an algebraic extension of $E_{j}$. We will see that comparing the local behavior of solutions of the corresponding differential equations at $p_{0}^{i}$ will yield a contradiction.

A result of Kolchin (Theorem of [8] or Exercise 8, Chapter V.23 of [9]) implies that there are indices $i \neq j$ and a homomorphism (defined over $C$ ) $f: G_{i} \rightarrow G_{j} / Z\left(G_{j}\right)$, where $Z\left(G_{j}\right.$ ) is the center of $G_{j}$, such that for every $h=\left(h_{1}, \ldots, h_{m}\right) \in G, f\left(h_{i}\right)=\pi\left(h_{j}\right)$, where $\pi$ is the canonical homomorphism $G_{i} \rightarrow G_{j} / Z\left(G_{j}\right)$. Note that since $G_{i}$ and $G_{j}$ are simple, the kernels of $f$ and $\pi$ are finite.

We now apply the maps $f$ and $\pi$ to the element $g=\left(g_{1}, \ldots, g_{m}\right) \in G^{0}(E)$ defined above. Since $f\left(g_{i}\right)=\pi\left(g_{j}\right)$, we have that $E_{i}$ and $E_{j}$ share the common subfield $K\left(f\left(g_{i}\right)\right)=$ $K\left(\pi\left(g_{j}\right)\right)$. Furthermore, $E_{i}$ and $E_{j}$ are algebraic extensions of this field since the kernels of $f$ and $\pi$ are finite. Therefore $E_{i}$ is contained in an algebraic extension of $E_{j}$.

By construction, $Y^{\prime}=A_{j} Y$ is not singular at $p_{0}^{i}$ and so the solutions of $Y^{\prime}=A_{j} Y$ at $p_{0}^{i}$ lie in $C((t))$ where $t$ is the local parameter at $p_{0}^{i}$. Therefore we can embedd $E_{j}$ into $C((t))$. This implies that $Y^{\prime}=A_{i} Y$ has a fundamental set of solutions in an algebraic extension of $C((t))$ and so must be regular singular at this point ([16], Exercise 3.29). This contradicts the fact that $Y^{\prime}=A_{i} Y$ has nonzero slopes at this point. This contradiction invalidates our 
assumption that $G^{\prime} \neq G$ and so the Galois group of $Y^{\prime}=A Y$ is $G$.

\section{An Alternate Construction for Finite Extensions of $\mathrm{SL}_{2}$}

In this section we present an alternate method for constructing linear differential equations whose Galois groups are finite extensions of $\mathrm{SL}_{2}$. In the previous sections, we considered groups of the form $H \ltimes G^{0}, H$ a finite group and $G^{0}$ of the type considered above, and showed that for any realization of $H$ as a Galois group of an extension $K$ of $C(x)$, we could find an equivariant $A$ such that $Y^{\prime}=A Y$ had Galois group $G^{0}$ over $K$. The construction described here begins by constructing a suitable $K$ and so does not work over any such $K$. On the other hand, it introduces fewer singularities and uses group theoretic facts that may be of independent interest. This construction was motivated by the Example on page 42 of [4].

We begin with a modification of a result of Borel and Serre ( 2], Lemma 5.11; c.f., 19], Lemma 10.10). For any algebraic group $G$ we define Int : $G \rightarrow \operatorname{Aut}\left(G^{0}\right)$ to be the map that sends an element to the automorphism resulting from conjugation by that element.

Lemma 5.1 Let $G$ be a linear algebraic group, $B$ a Borel subgroup of $G$ and $T$ a maximal torus of $B$.

1. There exists a finite subgroup $W$ of $G$ such that $W$ normalizes $B$ and $T$ and the natural projection $W \rightarrow G / G^{0}$ is surjective.

2. If, in addition, $G^{0}$ is semisimple and all automorphisms of $G^{0}$ are inner, then $\operatorname{Int}(W) \subset$ $\operatorname{Int}(T)$ and so $\operatorname{Int}(W)$ is a finite abelian group. If $G^{0}=\mathrm{SL}_{2}$ or $\mathrm{PSL}_{2}$, then $\operatorname{Int}(W)$ is cyclic.

Proof. 1. Let $N_{G}(B)$ be the normalizer of $B$ in $G$ and $N_{G}(B, T)$ be the subgroup of elements of $N_{G}(B)$ that normalize $T$ as well. Since all Borel subgroups of $G$ lie in $G^{0}$ and are conjugate in $G^{0}$ ([6], Theorem 21.3), we have that for any $g \in G$ there exists an $h \in G^{0}$ such that $g B g^{-1}=h B h^{-1}$. Therefore $h^{-1} g \in N_{G}(B)$ and we can conclude that $G=N_{G}(B) \cdot G^{0}$. Using the fact that the maximal tori of $B$ are all conjugate in $B$ (Theorem 19.3, 6]), we also have that $N_{G}(B)=N_{G}(B, T) \cdot B$. Lemma 10.10 of [19] implies that there exists a finite subgroup $W$ of $N_{G}(B, T)$ such that the natural projection $W \rightarrow N_{G}(B, T) / N_{G}(B, T)^{0}$ is surjective. We then have that the projection $W \rightarrow G / G^{0}$ is surjective as well.

2. (c.f., the proof of Theorem 27.4 in [6]) Since all automorphisms of $G^{0}$ are inner, for any element $w \in W$ there is an element $h \in G^{0}$ such that for all $g \in G^{0}, w g w^{-1}=h g h^{-1}$. Since $w$ normalizes $B$ and $T$, we have that $\operatorname{Int}(W) \subset \operatorname{Int}\left(N_{G^{0}}(B, T)\right)$. Since $B$ is a Borel 
subgroup, we have that $N_{G^{0}}(B, T) \subset N_{G^{0}}(B)=B$. An element of $B$ that normalizes $T$ must lie in $T$ (Proposition 19.4, Corollary 26.2A of [6]) so $\operatorname{Int}(W) \subset \operatorname{Int}(T)$. The final statement follows from the fact that a maximal torus of these groups has dimension 1.

Let $G$ be a linear algebraic group with $G^{0}$ semisimple and let $\mathcal{G}$ be its Lie algebra. If $T$ is a maximal torus of $G^{0}$, then its Lie algebra $\mathcal{T}$ is a Cartan subalgebra of $\mathcal{G}$ and we can decompose

$$
\mathcal{G}=\mathcal{T} \oplus \prod_{\alpha \in \Phi} \mathcal{G}_{\alpha}
$$

where $\Phi$ are the roots of $\mathcal{G}$ which we consider as multiplicative characters on $T$. If $W$ is the finite group described in Corollary [5.1] then for any $\alpha \in \Phi$ and $w \in W$ we define $\alpha(w)=\alpha(t)$ for any $t \in T$ such that $\operatorname{Int}(w)=\operatorname{Int}(t)$. Since the elements of $\Phi$ factor through Int : $G \rightarrow \operatorname{Aut}(G)$, each root in this way defines a multiplicative character on $W$.

Example 5.2 Let $G^{0}=\mathrm{SL}_{2}$ and assume that $T$ is the subgroup of diagonal matrices. As usual we let

$$
h=\left(\begin{array}{cc}
1 & 0 \\
0 & -1
\end{array}\right), \quad e=\left(\begin{array}{ll}
0 & 1 \\
0 & 0
\end{array}\right), \quad f=\left(\begin{array}{ll}
0 & 0 \\
1 & 0
\end{array}\right) .
$$

$\mathcal{T}$ is spanned by $h$ and there are two roots $\alpha$ and $-\alpha$ with $\mathcal{G}_{\alpha}$ being spanned by $e$ and $\mathcal{G}_{-\alpha}$ by $f$. Furthermore, considering the roots as characters on $T$, we have that

$$
\alpha\left(\begin{array}{cc}
a & 0 \\
0 & a^{-1}
\end{array}\right)=a^{2}, \quad-\alpha\left(\begin{array}{cc}
a & 0 \\
0 & a^{-1}
\end{array}\right)=a^{-2}
$$

Let $G=\mathrm{SL}_{2} \ltimes\{1,-1\}$ where the action of -1 on $\mathrm{SL}_{2}$ is given by conjugation by the matrix

$$
\left(\begin{array}{cc}
\sqrt{-1} & 0 \\
0 & -\sqrt{-1}
\end{array}\right) \text {. }
$$

We then have that $\operatorname{Int}(W)=\operatorname{Int}(H)$ where $W=\{1,-1\}$ and $H$ is the order four cyclic subgroup of $\mathrm{SL}_{2}$ generated by the above matrix. Note that $\alpha$ can be considered as the character on $W$ given by $\alpha(-1)=-1$.

Let $G$ be an algebraic group with $G^{0}=\mathrm{SL}_{2}$ and let $W$ be as in Lemma 5.1. We shall construct a differential equation having $W \ltimes G^{0}$ as its Galois group over $C(x)$. The group $G$ will then be the Galois group of a subfield $\tilde{E}$ of the Picard-Vessiot extension $E$ of $C(x)$ corresponding to this former equation.

We now use the notation $G$ to denote the group $\mathrm{SL}_{2} \ltimes W$ and $\mathcal{G}$ to denote the Lie algebra of $G$. Conjugation by an element of $W$ induces an automorphism of $G$ and also an automorphism of $\mathcal{G}$, which we again denote by conjugation. If $K$ is any field containing $C$ and $X=a h+b e+c f \in \mathcal{G}(K), a, b, c \in K$, then, for $w \in W$

$$
w^{-1} X w=a h+b \alpha\left(w^{-1}\right) e+c\left(-\alpha\left(w^{-1}\right) f\right)
$$


Note that if the image of $W$ in $\operatorname{Aut}(G)$ has order $n$, then $\alpha$ maps $W$ onto the group of $n^{\text {th }}$ roots of unity. We identfy this with the Galois group of $C\left(x, x^{1 / n}\right)$. Let $K$ be a Galois extension of $C(x)$ with Galois group $W$ such that the fixed field of the kernel of $\alpha$ is $C\left(x, x^{1 / n}\right), x^{\prime}=1$ and the action of $W$ on this latter field is given by $\alpha$. Theorem 7.13 of [18] implies that such a field exists. Let

$$
\tilde{A}=x^{-1 / n} e+x^{1 / n} f+x^{2} h .
$$

To construct a differential equation whose Galois group is $G$, Proposition 5.2 of [14] implies that it is enough to prove the following proposition.

Proposition 5.3 $\tilde{A}$ is equivariant and the differential Galois group of $Y^{\prime}=\tilde{A} Y$ over $C\left(x, x^{1 / n}\right)$ is $\mathrm{SL}_{2}$.

Proof. To prove the claim about the Galois group, we make a change of variables $x=z^{n}$. We then get a new equation $\frac{d Y}{d z}=A Y$ where

$$
A=n\left(z^{n-2} e+z^{n} f+z^{3 n-1} h\right)
$$

We will use the techniques of [13] to show that $\frac{d Y}{d z}=A Y$ has differential Galois group $\mathrm{SL}_{2}$ over $C(z)$.

Assuming that this latter fact is true, we claim that the differential Galois group of $Y^{\prime}=\tilde{A} Y$ over $C\left(x, x^{1 / n}\right)$ is $\mathrm{SL}_{2}$. To see this, let $K$ be a Picard-Vessiot extension of $C(z)=C\left(x^{1 / n}\right)$ for $\frac{d Y}{d z}=A Y$. Since $K$ has no new $\frac{d}{d z}$-constants, it has no new $\frac{d}{d x}$-constants. Furthermore, since the elements of $\mathrm{SL}_{2}$ commute with $\frac{d}{d z}$ and leave $C(z)$ fixed, they will commute with $\frac{d}{d x}=\frac{1}{n z^{n-1}} \frac{d}{d z}$. Therefore, $\mathrm{SL}_{2}$ is a subgroup of the differential Galois group $G$ of $K / C(z)$ with respect to $\frac{d}{d x}$. From the form of $\tilde{A}$, we see that $G \subset \mathrm{SL}_{2}$, so the claim is proved.

We now proceed to show that $\frac{d Y}{d z}=A Y$ has differential Galois group $\mathrm{SL}_{2}$ over $C(z)$. Since $C^{2}$ is a Chevalley module for $\mathrm{SL}_{2}$, Lemma 3.3 of [13] implies that it is enough to show that if

$$
c=c_{3 n-1} z^{3 n-1}+\ldots+c_{0} \in C[z] \text { and } w=w_{m} z^{m}+\ldots+w_{0} \in C^{2} \otimes C[z]
$$

and

$$
w^{\prime}-n\left[z^{n-2} e+z^{n} f+z^{3 n-1} h-c I\right] w=0
$$

then $w=0$.

To simplify notation, we let $u=\left(\begin{array}{l}1 \\ 0\end{array}\right)$ and $v=\left(\begin{array}{l}0 \\ 1\end{array}\right)$. These generate the root spaces of $\mathrm{SL}_{2}$ and $e u=0, e v=u, f u=v$, and $f v=0$ and that $u, v$ are eigenvectors of $h$. The proof 
of the above fact proceeds by considering the coefficients of powers of $z$ in equation (44). The highest power of $z$ that can appear is $z^{3 n-1+m}$ and its coefficient is

$$
n\left(c_{3 n-1} I-h\right) w_{m}=0 .
$$

We therefore have that $w_{m}$ is an eigenvector of $h$ and so we can assume that $w_{m}=$ $u, c_{3 n-1}=1$ or $w_{m}=v, c_{3 n-1}=-1$. Let us assume that $w_{m}=u$ and $c_{3 n-1}=1$. We shall write $w=p u+q v$ where $p, q \in C[z], p=z^{m}+$ lower degree terms, and $q=$ a polynomial of degree at most $m-1$. Substituting $w=p u+q v$ into equation (44), we have:

$$
\left(p^{\prime}-n z^{n-2} q-n z^{3 n-1} p+n c p\right) u+\left(q^{\prime}-n z^{n} p+n z^{3 n-1} q+n q c\right) v=0
$$

and therefore

$$
\begin{aligned}
p^{\prime}-n\left[z^{3 n-1}-c\right] p & =n z^{n-2} q \\
q^{\prime}+n\left[z^{3 n-1}+c\right] q & =n z^{n} p
\end{aligned}
$$

The right hand side of equation (6) has degree $n+m$. Since $z^{3 n-1}+c$ has degree $3 n-1$, $q$ must have degree $m-2 n+1$. Therefore the right hand side of equation (5) has degree $m-n-1$, while the degree of $\left[z^{2 n-1}-c\right] p$ is at least $m$ if $z^{3 n-1}-c \neq 0$. Therefore we have $c=z^{3 n-1}$ and that $p^{\prime}=n z^{n-2} q$. Comparing degress in this last equation, we have $m-1=n-2+m-2 n+1=m-n-1$ so $n=0$, a contradiction, unless $w=0$. If $w_{m}=v$ and $c_{3 m-1}=-1$ one argues in a similar way to also show that $w=0$.

\section{References}

[1] D. G. Babbitt and V. S. Varadarajan. Formal reduction of meromorphic differential equations: a group theoretic view. Pacific J. Math., 109(1):1-80, 1983.

[2] A. Borel and J.-P. Serre. Théorèmes de finitude en cohomologie galoisienne. Comment. Math. Helv., 39:111-164, 1964-65.

[3] N. Bourbaki. Groupes et Algèbres de Lie, Chaps. Y7 and 8. Masson, Paris, 1990.

[4] J. Hartmann. On the inverse problem in differential Galois theory. Thesis, University of Heidelberg, 2002 (available at www.ub.uni-heidelberg.de/archiv/3085).

[5] E. Hrushovski. Computing the Galois group of a linear differential equation. In Differential Galois Theory, volume 58 of Banach Center Publications, pages 97-138. Institute of Mathematics, Polish Academy of Sciences, Warszawa, 2002.

[6] J. Humphreys. Linear Algebraic Groups. Graduate Texts in Mathematics. SpringerVerlag, New York, 1975. 
[7] N. Katz. On the calculation of some differential Galois groups. Inventiones Mathematicae, 87:13-61, 1987.

[8] E. R. Kolchin. Algebraic groups and algebraic dependence. American Journal of Mathematics, 90:1151-1164, 1968.

[9] E. R. Kolchin. Differential Algebra and Algebraic Groups. Academic Press, New York, 1976.

[10] J. Kovacic. The inverse problem in the Galois theory of differential fields. Annals of Mathematics, 89:583-608, 1969.

[11] J. Kovacic. On the inverse problem in the Galois theory of differential fields. Annals of Mathematics, 93:269-284, 1971.

[12] S. Lang. Algebra. Addison Wesley, New York, 3rd edition, 1993.

[13] C. Mitschi and M. F. Singer. Connected linear groups as differential Galois groups. Journal of Algebra, 184:333-361, 1996.

[14] C. Mitschi and M. F. Singer. Solvable-by-finite groups as differential Galois groups. Ann. Fac. Sci. Toulouse, XI(3):403 - 423, 2002.

[15] T. Oberlies. PhD thesis, University of Heidelberg, 2003.

[16] M. van der Put and M.F. Singer. Galois Theory of Linear Differential Equations, volume 328 of Grundlehren der mathematischen Wissenshaften. Springer, Heidelberg, 2003.

[17] M. F. Singer. Moduli of linear differential equations on the Riemann sphere with fixed Galois group. Pacific Journal of Mathematics, 106(2):343-395, 1993.

[18] H. Volklein. Groups as Galois Groups, volume 53 of Cambridge Studies in Advanced Mathematics. Cambridge University Press, Cambridge, 1996.

[19] B. A. F. Wehrfritz. Infinite Linear Groups. Ergebnisse der Mathematik. SpringerVerlag, Berlin, 1973. 